\title{
ARTíCULO \\ Edad y crecimiento del sábalo Megalops atlanticus en la costa de Campeche, México
}

\author{
Age and growth of tarpon Megalops atlanticus in Campeche coast, Mexico
}

\section{Brenda Vega-Rodríguez y Luis Ayala-Pérez ${ }^{2}$}

\begin{abstract}
${ }^{1}$ Doctorado en Ciencias Biológicas y de la Salud, Universidad Autónoma Metropolitana, Unidad Xochimilco, Calzada del Hueso 1100, Col. Villa Quietud Delegación Coyoacán, México, D.F. C.P. 04960, México. bilanvr@ hotmail.com ${ }^{2}$ Departamento El Hombre y su Ambiente. Universidad Autónoma Metropolitana, Unidad Xochimilco, Calzada del Hueso 1100, Col. Villa Quietud Delegación Coyoacán, México, D.F. C.P. 04960, México
\end{abstract}

\begin{abstract}
Tarpon age and growth studies are scarce and most of them had some problems to determine age through annular marks on otoliths. The objective of this paper is to determine age and describe growth of tarpon in Campeche coast. Tarpons were collected between May 2009 and February 2012. Organisms were measured on fork length (Lf) and total weight $(\mathrm{Pt})$ to determine length-weight relationship. With simple regression we determined the relationship between transformed $\log _{10}$ of otolith length (Lot) and Lf. Age was established through growth rings on otoliths (sagitta). Growth parameters were calculated with an actual age-mean length key. Length and weight range was 15.3 to $191 \mathrm{~cm}$ of Lf and 0.028 to $57 \mathrm{~kg}$, respectively. Equation of length-weight relationship was $\mathrm{Pt}=1 \times 10^{-5} \mathrm{Lf} \mathrm{f}^{2.9782}$ with an allometric growth. Lot and Lf relationship equation was $\log _{10} \mathrm{Lf}=1.3752 \log _{10}$ Lot +0.2688 . Maximum age recorded was 60 years of an organism of 191 $\mathrm{cm}$ of $\mathrm{Lf}$. Von Bertalanffy growth model was expressed as $L f=183.9\left[1-\mathrm{e}^{-0.1(t+1.49)}\right]$. Maximum age of tarpon is still unknown; thereby it is necessary to incorporate precautionary principle on management strategies.
\end{abstract}

Key words: Length-weight relationship, allometry coefficient, otoliths, growth rings, von Bertalanffy model

Resumen.- Los estudios sobre la edad y el crecimiento del sábalo son escasos y la mayoría han tenido problemas para determinar la edad a través de marcas anulares en otolitos. El objetivo de este trabajo fue determinar la edad y describir el crecimiento del sábalo en la costa de Campeche. La recolección de organismos se realizó entre mayo 2009 y febrero de 2012. Se midió la longitud furcal (Lf) y el peso total (Pt) de los ejemplares para determinar su relación talla-peso. A partir de una regresión lineal se determinó la relación entre $\log _{10}$ de la longitud del otolito (Lot) y $\log _{10}$ de Lf. La edad se estableció mediante el conteo de anillos de crecimiento en otolitos (sagitta). Los parámetros de crecimiento se calcularon en base a una clave edad actual-longitud media. El intervalo de longitud y peso fue de 15,3 a $191 \mathrm{~cm}$ y 0,028 a $57 \mathrm{~kg}$, respectivamente. La ecuación talla-peso obtenida fue $\mathrm{Pt}=1 \times 10^{-5} \mathrm{Lf}$ 2,9782 con un crecimiento alométrico. La relación entre Lot y Lf fue $\mathrm{Log}_{10} \mathrm{Lf}=$ 1,3752 $\log _{10}$ Lot + 0,2688. La edad máxima registrada fue de 60 años en un organismo de $191 \mathrm{~cm}$ de Lf. El modelo de crecimiento de von Bertalanffy se expresó como $\mathrm{Lf}=183,9\left[1-\mathrm{e}^{-0,1(t+1,49)}\right]$. La edad máxima del sábalo aún se desconoce, por lo tanto se recomienda aplicar el principio precautorio dentro de las estrategias de aprovechamiento.

Palabras clave: Relación talla-peso, coeficiente de alometría, otolitos, anillos de crecimiento, modelo de Von Bertalanffy

\section{INTRODUCCIÓN}

Megalops atlanticus (Cuvier \& Valenciennes, 1847), comúnmente conocido como sábalo, es un pez que se distribuye en las aguas tropicales y subtropicales del océano Atlántico; desde Mauritania hasta Angola al este y desde Virginia hasta Brasil al oeste (Zale \& Merrifield 1989, Anyanwu \& Kusemiju 2008). En la zona costera de Costa Rica, Florida, Luisiana y México es un recurso económicamente importante que mantiene la pesca recreativa (Crabtree $e t$ al. 1995, Chacón 1997, Dailey et al. 2008). La pesca comercial de esta especie es ilegal en México y está destinada exclusivamente para la pesca deportiva-recreativa (Diario Oficial de la Federación 2009) $)^{1}$. En Tamaulipas, Veracruz y Campeche se realizan torneos de pesca de sábalo y desde hace algunos años se le ha promovido como un atractivo turístico en este último lugar.

${ }^{1}$ Diario Oficial de la Federación. 2009. Proyecto de modificación a la Norma Oficial Mexicana NOM-017-Pesc-1994, para regular las actividades de pesca deportivo-recreativa en las aguas de jurisdicción federal de los Estados Unidos Mexicanos. Secretaría de Gobernación, México. <dof.gob.mx/nota_detalle.php?codigo=5090557\&fecha=15/05/2009> 
La biología del sábalo es compleja, pues habita en diferentes ambientes durante su desarrollo. Los huevos son desovados en mar abierto y las larvas leptocéfalas inician su metamorfosis cuando se encuentran en la costa (Smith 1980, Zerbi et al. 2001). Los juveniles tempranos viven en estanques de elevada turbidez, someros, salobres y con bajas concentraciones de oxígeno (Rickards 1968, Chacón \& McLarney 1992). Mientras que los juveniles tardíos pueden localizarse en zonas de manglar, arroyos, canales, lagunas, estuarios y aguas costeras (Zale \& Merrifield 1989, Anyanwu \& Kusemiju 2008). Los sábalos adultos habitan principalmente en la zona costera, aunque también pueden observarse en mar abierto (Ault et al. 2008).

Los estudios sobre edad y crecimiento del $M$. atlanticus son escasos a pesar de su popularidad y amplia distribución mundial. Esta situación se debe, en parte, a la dificultad para capturar ejemplares. En investigaciones previas se han obtenido sábalos a partir de torneos, taxidermistas y mediante el empleo de varias artes de pesca (Cyr 1991, Crabtree et al. 1995, Crabtree et al. 1997). Por otro lado, la estimación de edad a partir del conteo de anillos de crecimiento en otolitos ha presentado complicaciones en esta especie. Por ejemplo, Crabtree et al. (1995) tuvieron dificultades para interpretar algunas líneas de crecimiento y posteriormente Crabtree et al. (1997) descartaron más de la mitad de los otolitos pues no observaron características definidas en ellos.

La información sobre la edad y crecimiento del sábalo es fundamental para implementar medidas regulatorias de pesca deportiva en México, en donde tiene un estatus con potencial de desarrollo (Diario Oficial de la Federación 2012) $)^{2}$. Este estudio, para contribuir con conocimiento científico y pesquero, determinó la edad y crecimiento del M. atlanticus mediante anillos en otolitos de organismos capturados en la costa de Campeche.

\section{Materiales y MÉTODOS}

El área de estudio se localizó en las coordenadas $18^{\circ} 12^{\prime}-$ $20^{\circ} 51^{\prime} \mathrm{N}$ y $90^{\circ} 24^{\prime}-92^{\circ} 18^{\prime} \mathrm{W}$ del estado de Campeche, México (Fig. 1). La región se divide en 2 zonas de acuerdo a sus características hidrológicas. En la primera se ubica el Área de Protección de Flora y Fauna 'Laguna de Términos', en donde se encuentran 4 sistemas fluvio-lagunares (PomAtasta, Palizada-Del Este, Chumpán-Balchacah, y Candelaria-Panlau) que desembocan en la laguna citada.
Además este complejo tiene un amplio mosaico de vegetación de pantano, manglares y pastos marinos (INE 1997). En la segunda zona se encuentra la Reserva de la Biosfera 'Los Petenes' que se caracteriza por integrar un conjunto de islas de vegetación (manglar) asociadas a resurgencias de agua dulce, además en el área de influencia marina se pueden observar comunidades de pastos marinos (Palacio-Aponte et al. 2005, CONANP 2006).

La recolección de ejemplares se realizó desde mayo de 2009 a febrero de 2012 en la marisma Laguna Larga, río Mameyal, zona costera y en mar abierto (Fig. 1). En la Tabla 1 se observan las diferentes fuentes de donde se obtuvieron los sábalos. Además, se registraron biometrías de 21 peces, 13 provinieron de sábalos que se vendían en el mercado (se consiguieron los otolitos de 3 individuos), 2 fueron de organismos liberados en un torneo y 6 fueron proporcionados por pescadores recreativos.

Se registraron las biometrías de longitud furcal ( $\mathrm{Lf}$ ) y peso total $(\mathrm{Pt})$, expresado en $\mathrm{cm}$ y $\mathrm{kg}$ respectivamente. A partir de estas variables se calculó la relación talla-peso (Ricker 1975):

$$
P t=a L f^{b}
$$

donde $a$ es el intercepto y $b$ es la pendiente o el coeficiente de alometría. La significancia estadística del coeficiente de alometría respecto al valor de tres se determinó mediante una prueba $t$ de Student (Zar 1999).

El procesamiento de los otolitos (sagitta) se basó en la técnica de inclusión en resina epóxica (Secor et al. 1992). Los bloques de plástico con los otolitos embebidos fueron pulidos, en la cara interna del plano sagital, con una rectificadora marca Dremel 300 con discos para lijar número 411. Posteriormente la pieza fue pulida manualmente en húmedo con una lija marca Fandeli A-99. Las lecturas se realizaron a ojo desnudo a partir de fotografías tomadas con una cámara digital VE-MC3 montada en un microscopio estereoscópico con luz transmitida en diferentes aumentos (1 a 10x), de esta manera se facilitó el conteo de las marcas opacas en los otolitos.

Se registró la distancia del rostrum al postrostrum para establecer la longitud del otolito (Lot) en mm con una regla Vernier (Fig. 2). La función potencial que describe la relación entre Lot y Lf fue ajustada a una regresión lineal mediante la transformación logaritmo en base 10 de ambas variables.

${ }^{2}$ Diario Oficial de la Federación. 2012. Acuerdo mediante el cual se da a conocer la actualización de la Carta Nacional Pesquera. Secretaría de Gobernación, México. <www.dof.gob.mx/nota_detalle_popup.php?codigo=5265388> 


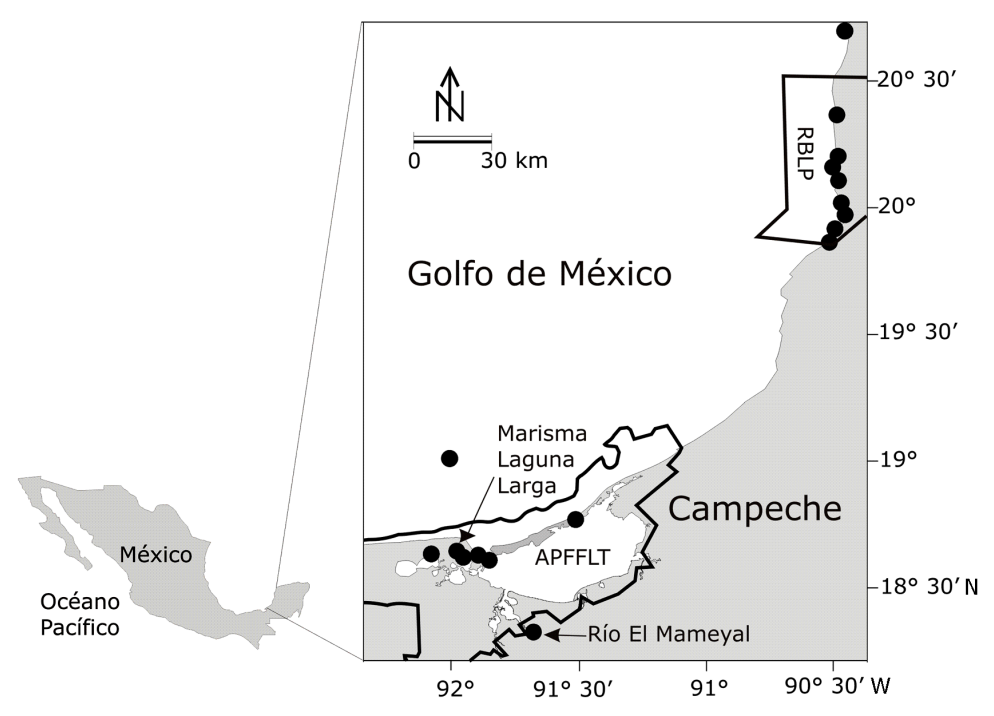

Figura 1. Área de estudio en la costa de Campeche. Líneas gruesas indican los límites del Área de Protección de Flora y Fauna 'Laguna de Términos' (APFFLT) y de la Reserva de la Biosfera 'Los Petenes' (RBLP); círculos negros señalan los sitios de captura / Study area on Campeche coast. Thick lines indicate the limits of the natural protected areas 'Laguna de Terminos' (APFFLT) and 'Los Petenes' (RBLP); black circles show catch sites

Tabla 1. Fuentes y métodos de obtención de los sábalos. $C=$ caña; $R E=$ red de enmalle experimental $(100 \mathrm{~m} \times 2,5$ $\mathrm{cm}$, con luz de malla de 2,5, 5,08 y 10,16 cm); $A=$ atarraya ( $2 \mathrm{~m}$ de diámetro y $1,5 \mathrm{~cm}$ de luz de malla); * algunas veces se comercializa en el mercado cuando el sábalo es capturado como fauna de acompañamiento; $\mathrm{SI}=\sin$ información / Sources where tarpons were caught. $\mathrm{C}=$ hook and line gear; $\mathrm{RE}=$ gillnet $(100 \mathrm{~m} \times 2.5 \mathrm{~cm}$, mesh size of 2.5, 5.08 y $10.16 \mathrm{~cm}) ; A=$ castnet $(2 \mathrm{~m}$ diameter and mesh size of $1.5 \mathrm{~cm}) ;{ }^{*}$ sometimes is sold on the market when is caught as bycatch; $\mathrm{SI}=$ without information

\begin{tabular}{lccc}
\hline Fuente & Arte de pesca & Sitios & $\mathrm{n}$ \\
\hline Muestreos & C, RE y A & Costa, río Mameyal y marisma Laguna Larga & 85 \\
Torneos & C & Costa y mar abierto & 26 \\
Donaciones & C & Costa & 5 \\
Comprados & SI & SI & 4 \\
\hline
\end{tabular}

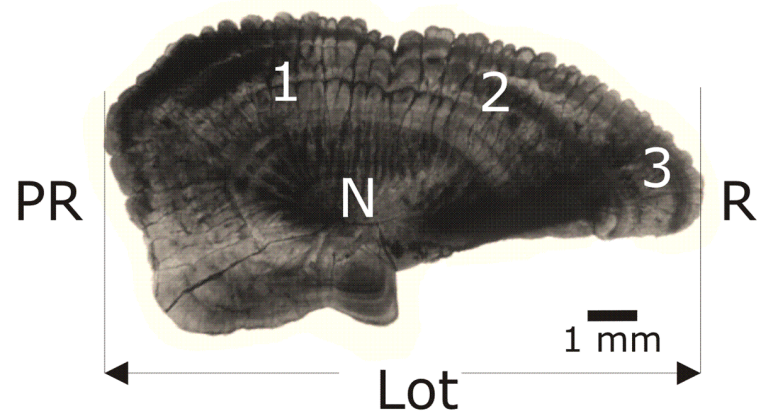

Figura 2. Otolito de un sábalo de 3 años de edad. $\mathbf{R}=$ rostrum; $\mathbf{P R}=$ postrostrum; $\mathbf{N}=$ núcleo; Lot= longitud del otolito / Otolith of a tarpon which was 3 years old. $R=$ rostrum; $\mathrm{PR}=$ postrostrum; $\mathrm{N}=$ nucleus; $\mathrm{Lot}=$ otolith length 
Las lecturas de las marcas opacas anuales (annulli) fueron realizadas por dos personas de manera independiente y la precisión del conteo de marcas anulares fue examinada mediante el Índice de Error Promedio Porcentual (Beamish \& Fournier 1981):

$$
E P P=\frac{1}{n} \sum_{j=1}^{n}\left[\frac{1}{R} \sum_{i=1}^{R} \frac{|x i j-x j|}{x j}\right] \times 100
$$

donde $n$ es el número de muestras, $R$ es el número de veces que se contaron los anillos de un organismo, xij es la edad $i$ determinada para el pez $j, x j$ es el promedio de las edades para el pez $j$.

La validación de los anillos de crecimiento mediante el análisis de incremento marginal no es recomendable para especies de larga vida como el sábalo, ya que los anillos se comprimen cerca del margen del otolito (Campana 2001, Choat et al. 2009). Por esta razón se asumió que la formación de anillos es anual, ya que Cyr (1991) y Crabtree et al. (1995) lo comprobaron mediante la técnica de marcaje químico (oxitetraciclina hidrocloruro).

Se elaboró una clave edad actual-longitud media con las longitudes de Lf de 122 ejemplares que mostraron annulli visibles, lo cual sirvió como base para estimar los parámetros de crecimiento que fueron calculados a partir de una regresión no lineal con la rutina de Salgado-Ugarte et al. (2005) en el programa Stata 7.0. La expresión de los resultados se realizó mediante el ajuste de la ecuación del modelo de crecimiento de Von Bertalanffy (1938):

$$
L f=L_{\infty}\left[1-\exp ^{\left(-k\left(t-t_{0}\right)\right.}\right]
$$

donde $L f$ es la longitud furcal a la edad $t, L_{\infty}$ es la longitud máxima asintótica, $k$ es la constante de crecimiento y $t_{0}$ es la edad de los peces cuando hipotéticamente tienen longitud cero.

\section{Resultados}

Se analizó un total de 141 biometrías de Lf y Pt, cuyo intervalo fue de 15,3 a $191 \mathrm{~cm} \mathrm{y} 0,028$ a $57 \mathrm{~kg}$, respectivamente. La frecuencia de tallas mostró una moda entre los 30-40 cm y otra más entre los 160-180 cm (Fig. 3).

La función talla-peso se definió como $\mathrm{Pt}=1 \times 10^{-5} \mathrm{Lf}^{2,9782}$ $\left(\mathrm{r}^{2}=0,9917, P<0,0001\right)$ (Fig. 4) y el coeficiente de alometría $(b=2,9782 \pm 0,023)$ fue significativamente diferente de 3 ( $t$-Student, $t=-6,7$, g.l.= 139, $P<0,0001$ ), por lo tanto el crecimiento de la muestra fue de tipo alométrico.

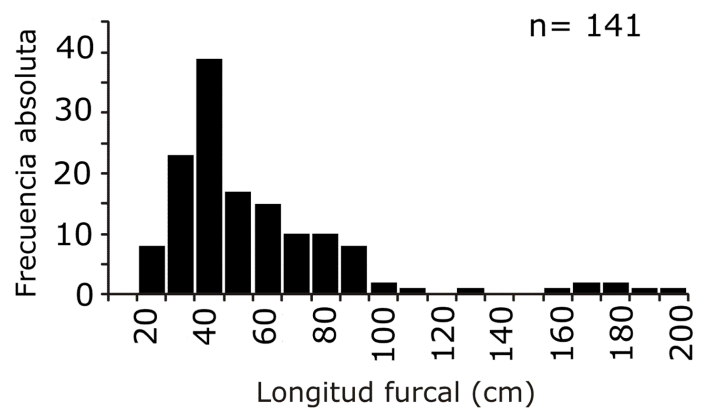

Figura 3. Frecuencia de tallas del M. atlanticus / Tarpon length frequency $M$. atlanticus

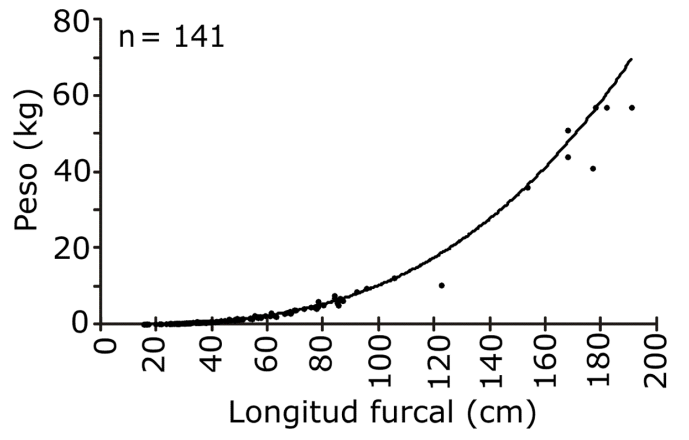

Figura 4. Relación entre la longitud furcal y el peso total del $M$. atlanticus / Fork length and total weight relationship of $M$. atlanticus

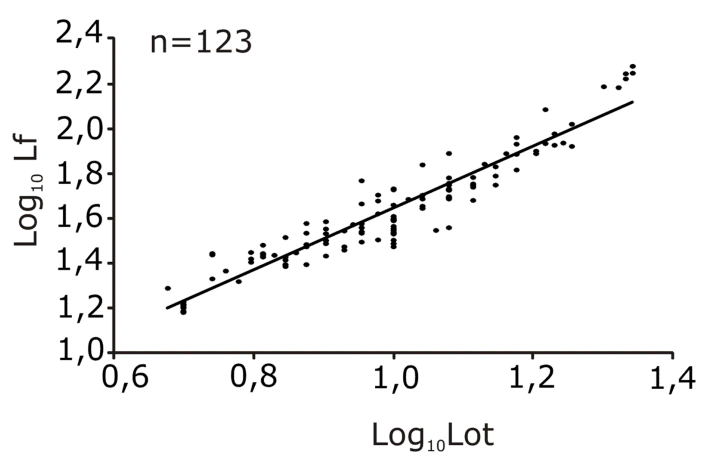

Figura 5. Regresión lineal entre la longitud del otolito $\left(\log _{10} \operatorname{Lot}\right)$ y la longitud furcal ( $\log _{10}$ Lf) / Linear regression between otolith length ( $\log _{10}$ Lot) and fork length $\left(\log _{10} L f\right)$ 

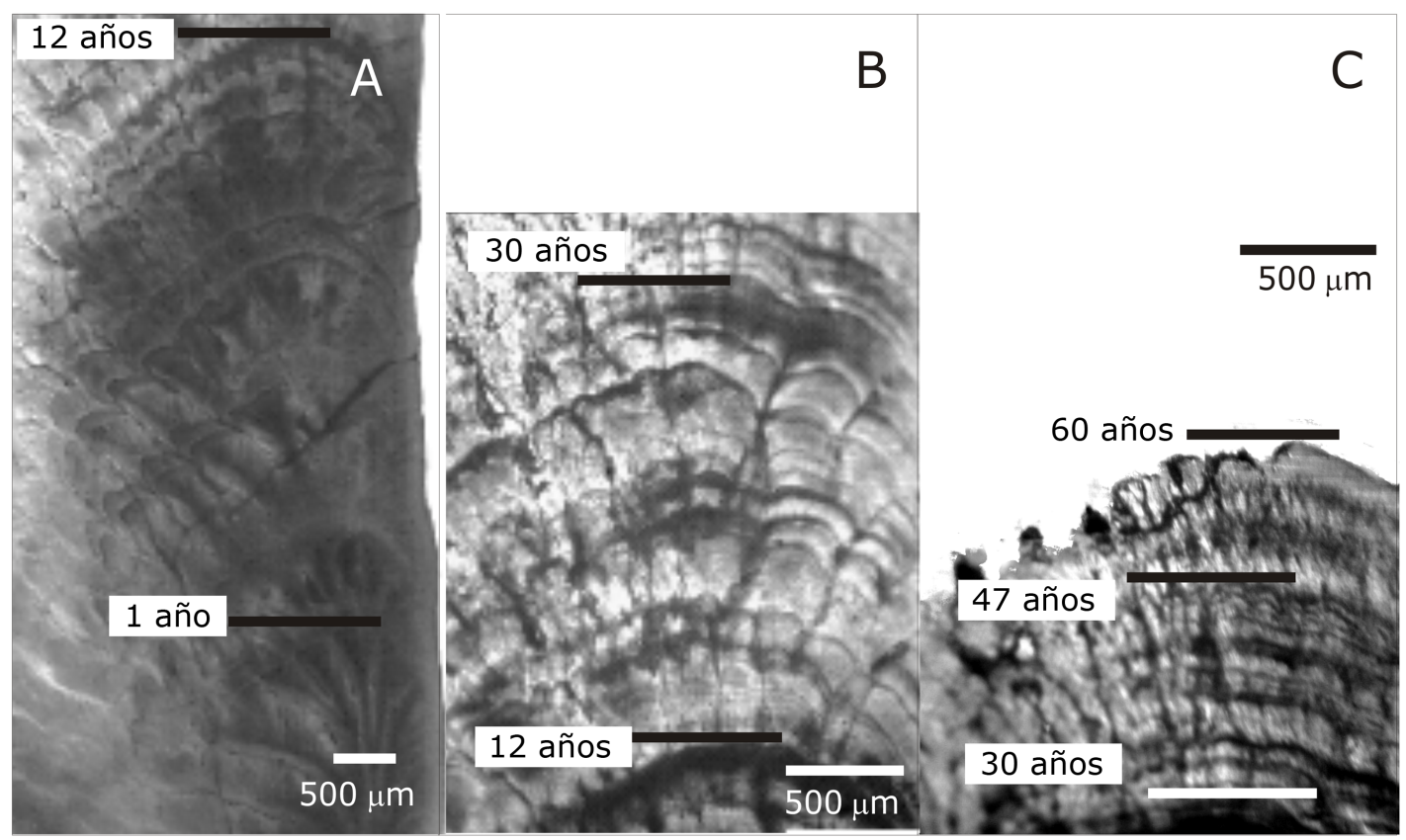

Figura 6. Sección sagital de un otolito de M. atlanticus que muestra líneas de crecimiento anual correspondientes a una edad de 60 años. (A) marcas de crecimiento desde un año a los 12 años; (B) marcas de crecimiento desde los 12 a los 30 años; (C) marcas de crecimiento desde los 30 a los 60 años / Sagittal sections of an otolith of M. atlanticus that shows annual growth lines corresponding to an age of 60 years. (A) growth marks since one year to 12 years; (B) growth marks since 12 to 30 years; (C) growth marks since 30 to 60 years

Tabla 2. Clave edad actual -longitud media. Los valores en paréntesis son valores estándar / Age- mean length key. Values in parentheses are standard errors

\begin{tabular}{ccc}
\hline $\begin{array}{c}\text { Grupo de } \\
\text { edad (años) }\end{array}$ & $\begin{array}{c}\text { Longitud } \\
\text { media }(\mathrm{cm})\end{array}$ & $\mathrm{n}$ \\
\hline 0 & $28,6(7,0)$ & 52 \\
1 & $39,8(6,4)$ & 24 \\
2 & $53,4(4,1)$ & 19 \\
3 & $63,7(8,6)$ & 9 \\
4 & $87,7(6,0)$ & 6 \\
5 & $88(2,9)$ & 4 \\
6 & 106 & 1 \\
10 & 122,5 & 1 \\
17 & 154 & 1 \\
18 & 168 & 1 \\
27 & 168 & 1 \\
31 & 177 & 1 \\
53 & 178 & 1 \\
60 & 191 & 1 \\
\hline
\end{tabular}

Se analizaron 123 otolitos, de los cuales, un organismo de 78,1 cm de Lf no presentó marcas visibles de crecimiento. La relación entre Lot y Lf se expresó como $\log _{10} \mathrm{Lf}=1,3752 \log _{10} \operatorname{Lot}+0,2688\left(\mathrm{r}^{2}=0,8938\right.$, g.l. $=121, P$ $<0,0001)$ (Fig. 5).

El valor de EEP (4,6\%) indicó una elevada reproducibilidad entre ambas lecturas, por tal motivo se utilizaron los conteos realizados por el primer lector. La edad máxima observada fue de 60 años en un sábalo de $191 \mathrm{~cm}$ de Lf (Fig. 6). En la Tabla 2 se muestra la clave edad actual- longitud media establecida, mientras que en la Tabla 3 se entregan los resultados derivados de la regresión no lineal. La ecuación de la curva de crecimiento se expresó como $\mathrm{Lf}=183,9\left[1-\mathrm{e}^{-0,1(\mathrm{t}+(-1,49))}\right]$ (Fig. 7).

\section{Discusión}

La distribución de frecuencia de tallas observada indicó que el $94 \%$ de la muestra estuvo integrada por organismos juveniles que se encuentran por debajo de la talla de primera madurez (Fig. 3); que de acuerdo con Ault et al. (2008) se alcanza a los $118 \mathrm{~cm}$ de Lf. Debe considerarse la posibilidad de que ciertos tamaños se encuentren más representados que otros debido a que en este estudio se utilizaron diferentes fuentes, lugares y artes de pesca. 
Tabla 3. Parámetros de crecimiento para $M$. atlanticus en la costa de Campeche. $L=$ longitud asintótica $(\mathrm{cm}) ; k=$ coeficiente de crecimiento; $t_{0}=$ longitud a la edad cero; los errores estándar, valores $P$, intervalos de confianza y correlaciones son aproximaciones asintóticas. El tamaño de muestra utilizado para el ajuste fue $n=14$ / Growth parameters for $M$. atlanticus in Campeche coast. $L_{\propto}=$ asymptotic length $(\mathrm{cm}) ; k=$ growth coefficient; $t_{0}=$ length at age zero; standard errors, $\mathrm{P}$ values, confidence intervals and correlations are asymptotic approach. Sample size used for adjustment was $n=14$

\begin{tabular}{|c|c|c|c|c|c|c|}
\hline \multirow{2}{*}{ Lf media } & \multirow{2}{*}{ Coeficiente } & \multirow{2}{*}{$\begin{array}{c}\text { Error } \\
\text { estándar }\end{array}$} & \multirow{2}{*}{ t-Student } & \multirow{2}{*}{$P>|\mathrm{t}|$} & \multicolumn{2}{|c|}{$\begin{array}{c}\text { Intervalo de } \\
\text { confianza 95\% }\end{array}$} \\
\hline & & & & & Inferior & Superior \\
\hline $\mathrm{L}_{\infty}(\mathrm{cm})$ & 183,9 & 3,29 & 55,82 & 0,0001 & 176,6 & 191,1 \\
\hline K & 0,1 & 0,007 & 13,03 & 0,0001 & 0,08 & 0,12 \\
\hline $\mathrm{t}_{0}$ (años) & $-1,49$ & 0,3 & $-4,94$ & 0,0002 & $-2,1$ & $-0,82$ \\
\hline
\end{tabular}

En investigaciones previas se han observado los siguientes coeficientes de alometría, $b=2,7$ (Ault et al. 2008), b=2,8 (Cyr 1991), b=2,9 (Crabtree et al. 1995) y b= 3,1 (Chacón 1993), sin mencionar el tipo de crecimiento. El intervalo que muestran los valores mencionados incluye al calculado en este estudio $(b=2,9782)$, el cual indicó que el sábalo tuvo un mayor incremento en la longitud que en el peso.

Cyr (1991) realizó una regresión potencial entre el peso del otolito y la longitud estándar $(a=7,96$ y b=0,591) y el ajuste que tuvieron sus datos al modelo fue del $84 \%$. Crabtree et al. (1997), mediante una regresión lineal entre $\log _{10}$ del peso del otolito y $\log _{10} \operatorname{Lf}(a=3,2861$ y $b=0,3386)$, tuvieron un ajuste del $62 \%$. En este estudio la relación entre $\log _{10}$ Lot y $\log _{10}$ Lf tuvieron un ajuste del $89 \%$ (Fig. $5)$, por lo tanto, la longitud del otolito también pude considerarse como un parámetro que permitiría predecir la edad.

La máxima longitud furcal reportada para el $M$. atlanticus es de $243 \mathrm{~cm}$ (Mc Clane 1974) y se estima que esta especie podría llegar a vivir más de 82 años (Burton et al. 1999). Crabtree et al. (1995) contaron un máximo de 55 años para una hembra de 204,5 cm de Lf en Florida; además mencionaron que tuvieron problemas para diferenciar líneas de crecimiento, pues algunas presentaban bifurcaciones. Posteriormente Andrews et al. (2001), reexaminaron esta muestra con una técnica radiométrica y estimaron que este organismo podría exceder los 78 años. La edad máxima estimada en el

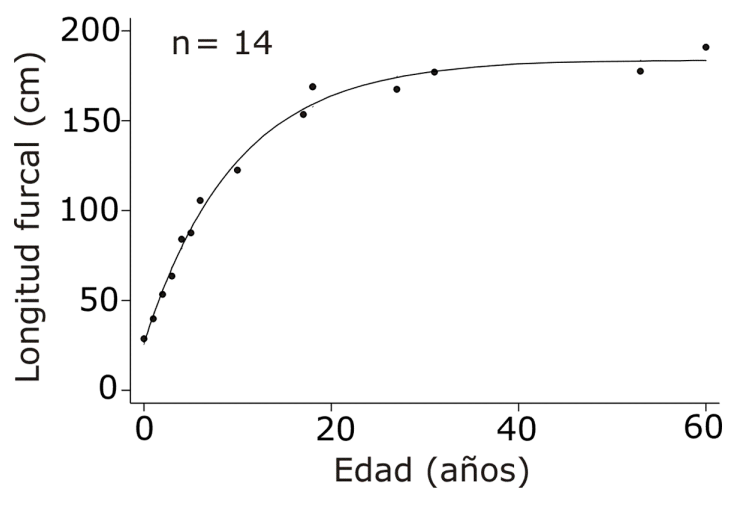

Figura 7. Curva de crecimiento en longitud furcal (función de Von Bertalanffy) para M. atlanticus en la costa de Campeche (círculos negros= valores observados; línea= valores ajustados) / Growth curve on fork length (von Bertalanffy function) for $M$. atlanticus on Campeche coast (black circles= observed values; line= adjusted values)

presente estudio fue de 60 años para un ejemplar de 191 $\mathrm{cm}$ de Lf y aunque no se observaron bifurcaciones, debe considerarse que el pulido pudo borrar algunos anillos que eran tenues o que se encontraban al borde del otolito. También es necesario destacar que Crabtree et al. (1995) utilizaron secciones transversales de los otolitos, mientras que en este estudio se analizaron en un plano sagital. El crecimiento de los otolitos no es uniforme y algunos no tienen ningún plano que contenga todos los incrementos (Cyr 1991, Secor et al. 1992). 
Por otro lado, Crabtree et al. (1997) registraron una edad máxima de 47 años en una hembra $(153 \mathrm{~cm})$ y un macho $(130 \mathrm{~cm})$ en Costa Rica, también observaron que los sábalos en esta región son más pequeños que en Florida. De acuerdo con Luo et al. (2008), muchos sábalos del stock de pesca en EEUU tienen amplios circuitos migratorios a lo largo de la costa del Golfo de México, por tal motivo, es probable que los organismos que habitan en Campeche alcancen tamaños y edades similares a los que han sido analizados en Florida.

En la Tabla 4 se presenta una comparación de los parámetros de crecimiento de $M$. atlanticus calculados en investigaciones previas. Las estimaciones de este estudio fueron similares a los presentados por Crabtree et al. (1995) y Ault et al. (2008) para hembras. Así mismo, en la Tabla 4 se distingue que los valores de $\mathrm{L}_{\propto}$ son inferiores a Lf máxima observada en cada investigación. Mathews \& Samuel (1990) demostraron que la longitud asintótica generalmente se subestima en especies longevas. Por otro lado, Andrews et al. (2001) publicaron la $\mathrm{L}_{\text {» }}$ más cercana al valor de Lf máxima registrada, sin embargo, aclaran que sus determinaciones pueden ser subjetivas debido al bajo número de muestras y a los amplios intervalos de confianza que utilizaron.

En conclusión, aún existe cierta incertidumbre en relación a las estimaciones de edad y de parámetros de crecimiento del M. atlanticus. Esto podría traer como consecuencia la sobreexplotación de la población del sábalo, tal como ha sucedido con el pez anaranjado (Hoplostethus atlanticus) ya que su edad fue subestimada y su tasa de crecimiento fue sobrestimada (Fenton et al. 1991, Smith et al. 1995). Aunque los análisis radiométricos han probado ser más efectivos son también costosos, se necesita un presupuesto de aproximadamente $\$ 1000$ dólares por grupo de edad (Campana 2001). Por esta razón, se recomienda aplicar el principio precautorio dentro de las estrategias de aprovechamiento de este recurso para asegurar su conservación (FAO 1999).

\section{Agradecimientos}

A CONACyT por financiar el proyecto CAMP2008C0195731 y por la beca de doctorado No. 35302. Además se agradece la valiosa colaboración del Club de Caza Pesca y Tiro 'Nelo Majarréz', a la Asociación. Estatal de Pesca Deportiva y Prestadores de Servicios Náuticos de Campeche, A.C., a Isabel González, Janeth Hernández, Mayrem Leal, Lucía Maura, Jonathan Nochebuena, Alberto Novelo, José Hernández, Miguel Benítez, Víctor Rodríguez y en especial a Bernabé Pastrana y a su familia por su apoyo en la captura de los peces.

Tabla 4. Comparación de los parámetros de crecimiento de Von Bertalanffy para M. atlanticus reportados en la literatura científica. Lf max= longitud furcal máxima $(\mathrm{cm}) ; \mathrm{L}_{\alpha}=$ longitud asintótica $(\mathrm{cm}) ; \mathrm{k}=$ coeficiente de crecimiento; $\mathrm{t}_{0}=$ longitud a la edad cero; $\mathrm{SI}=$ sin información / Comparison of von Bertalanffy growth parameters for $M$. atlanticus reported on scientific literature. Lf max= maximum length $(\mathrm{cm}) ; \mathrm{L}_{\alpha}=$ asymptotic length $(\mathrm{cm}) ; \mathrm{k}=$ growth coefficient; $\mathrm{t}_{0}=$ length at age zero; $\mathrm{SI}=$ without information

\begin{tabular}{llcccccc}
\hline Área & Grupo & Lf max & $\mathrm{L}_{\infty}$ & $\mathrm{k}$ & $\mathrm{t}_{0}$ & Método & Referencia \\
\hline \multirow{2}{*}{ Florida } & Todos & 188,0 & 164,7 & 0,102 & $-0,61$ & Anillos en otolitos & Cyr (1991) \\
& Hembras & 188,0 & 165,8 & 0,136 & 1,153 & & \\
& Machos & 168,0 & 148,0 & 0,114 & $-2,86$ & & \\
& & & & & & & \\
& Hembras & 204,5 & 181,8 & 0,103 & $-1,41$ & Anillos en otolitos & Crabtree et al. (1995) \\
& Machos & 188,4 & 156,7 & 0,123 & $-1,57$ & & \\
& Hembras & 204,5 & 203,0 & 0,080 & $-1,41$ & Análisis radiométricos & Andrews et al. (2001) \\
& Machos & 162,0 & 155,0 & 0,190 & $-1,33$ & & \\
& Hembras & SI & 181,5 & 0,102 & $-1,20$ & Anillos en otolitos & Ault et al. (2008) $)^{2}$ \\
& Machos & SI & 157,0 & 0,116 & $-1,51$ & & Este estudio \\
\hline
\end{tabular}

${ }^{1}$ valores basados en longitud estándar

${ }^{2}$ valores basados en una actualización del estudio de Crabtree et al. (1995) 


\section{LiTERATURA CITADA}

Andrews AH, EJ Burton, KH Coale, GM Cailliet \& RE Crabtree. 2001. Radiometric age validation of Atlantic tarpon, Megalops atlanticus. Fishery Bulletin 99(3): 389398.

Anyanwu PE \& K Kusemiju. 2008. The Nigerian tarpon: Resource ecology and fishery. In: Ault J (ed). Biology and management of the world tarpon and bonefish fisheries, pp. 115-128. CRC Press, Boca Raton.

Ault JS, R Humston, MF Larkin, E Perusquia, NA Farmer, J Luo, N Zurcher, SG Smith, LR Barbieri \& JM Posada. 2008. Population dynamics and resource ecology of Atlantic tarpon and bonefish. In: Ault J (ed). Biology and management of the world tarpon and bonefish fisheries, pp. 217-258. CRC Press, Boca Raton.

Beamish RJ \& DA Fournier. 1981. A method for comparing the precision of a set of age determinations. Canadian Journal of Fisheries and Aquatic Sciences 38(8): 982-983.

Burton EJ, AH Andrews, KH Coale \& GM Cailliet. 1999. Application of radiometric age determination to three longlived fishes using $210 \mathrm{~Pb}$ : 226Ra disequilibria in calcified structures: a review. In: Music J (ed). Life in the slow lane: ecology and conservation of long-lived marine animals: Proceedings of the Symposium Conservation of Long-Lived Marine Animals held at Monterey, California, USA 24 August 1997, pp. 77-88. American Fisheries Society, Maryland.

Campana SE. 2001. Accuracy, precision and quality control in age determination, including a review of the use and abuse of age validation methods. Journal of Fish Biology 59(2): 197-242.

Chacón CD. 1993. Biometrical aspects in a population of Megalops atlanticus (Pisces: Megalopidae). Revista de Biología Tropical 41(Suppl. 1): 13-18.

Chacón CD. 1997. Abundance and diversity of ichthyic resources used for recreational fishing in Barra del Colorado, Costa Rica. Revista de Biología Tropical 44-45(3-1): 489498.

Chacón CD \& WO McLarney. 1992. Early development of Megalops atlanticus (Pisces: Megalopidae). Revista de Biología Tropical 40(2): 171-178.

Choat JH, JP Kritzer \& JL Ackerman. 2009. Ageing in Coral Reef Fishes: Do we need to validate the periodicity of increment formation for every species of fish for which we collect age-based demographic data? In: Green B, B Mapstone, G Carlos \& G Begg (eds). Tropical fish otoliths: Information for assessment, management and ecology, pp. 23-54. Springer, New York.

CONANP. 2006. Programa de conservación y manejo Reserva de la Biosfera 'Los Petenes', 203 pp. Dirección General de Manejo para la Conservación, Comisión Nacional de Áreas Naturales Protegidas, México.
Crabtree RE, EC Cyr \& JM Dean. 1995. Age and growth of tarpon, Megalops atlanticus, from South Florida Waters. Fishery Bulletin 93(4): 619-628.

Crabtree RE, EC Cyr, CD Chacón, WO McLarney \& JM Dean. 1997. Reproduction of tarpon, Megalops atlanticus, from Florida and Costa Rican waters and notes on their age and growth. Bulletin of Marine Science 61(2): 271-285.

Cyr EC. 1991. Aspects of the life history of the tarpon Megalops atlanticus, from south Florida. Tesis Doctoral, Universidad de Carolina del Sur, Columbia, 138 pp.

Dailey W, AM Landry Jr \& L Kenyon II. 2008. The Louisiana recreational tarpon fishery. In: Ault $\mathbf{J}$ (ed). Biology and management of the world tarpon and bonefish fisheries, pp. 57-68. CRC Press, Boca Raton.

FAO. 1999. Precautionary approach to capture fisheries and species introductions, 54 pp. FAO Technical Guidelines for Responsible Fisheries, Roma.

Fenton GE, SA Short \& DA Ritz. 1991. Age determination of orange roughy, Hoplostethus atlanticus (Pisces: Trachichthyidae) using 210Pb:226Ra disequilibria. Marine Biology 109(2): 197-202.

INE. 1997. Programa de manejo del Área de Protección de Flora y Fauna 'Laguna de Términos', 167 pp. Instituto Nacional de Ecología, México.

Luo J, JS Ault, MF Larkin, R Humston \& DB Olson. 2008. Seasonal migratory patterns and vertical habitat utilization of Atlantic tarpon (Megalops atlanticus) from satellite PAT tags. In: Ault J (ed). Biology and management of the world tarpon and bonefish fisheries, pp. 275-299. CRC Press, Boca Raton.

Mathews CP \& M Samuel. 1990. The relationship between maximum and asymptotic length in fishes. Fishbyte 8(2): 14-16.

McClane A. 1974. McClane's New Standard Fishing Encyclopedia, 1156 pp. Holt, Rinehart and Winston, Nueva York.

Palacio-Aponte AG, V Medina-Medina \& F Bautista. 2005. Diagnóstico ambiental de la costa del estado de Campeche: Enfoques geomorfológico y geopedológico. En: BautistaZuñiga F \& A Palacio-Aponte (eds). Caracterización y manejo de los suelos de la Península de Yucatán: Implicaciones agropecuarias, forestales y ambientales, pp. 59-72. Universidad Autónoma de Campeche, Universidad Autónoma de Yucatán, Instituto Nacional de Ecología, México.

Rickards WL. 1968. Ecology and growth of juvenile tarpon, Megalops atlanticus, in a Georgia Salt Marsh. Bulletin of Marine Science 18(1): 220-239.

Ricker WE. 1975. Computation and interpretation of biological statistics of fish populations, Bulletin of Fisheries Research Board of Canada 191: 1-382. 
Salgado-Ugarte IH, JL Gómez-Márquez \& B PeñaMendoza. 2005. Métodos actualizados para análisis de datos biológico-pesqueros, 240 pp. Facultad de Estudios Superiores Zaragoza y Universidad Nacional Autónoma de México, México.

Secor DH, JM Dean \& EH Laban. 1992. Otolith removal and preparation for microstructural examination. In: Stevenson D \& S Campana (eds). Otolith microstructural examination and analysis, pp. 19-57. Canadian Special Publication Fisheries and Aquatic Sciences, Ottawa.

Smith DG. 1980. Early larvae of the tarpon, Megalops atlanticus Valeciennes, (Pisces Elopidae), with notes on spawning in the Gulf of Mexico and the Yucatan Channel. Bulletin of Marine Science 30(1): 136-141.

Smith DC, SG Robertson, GE Fenton \& SA Short. 1995. Age determination and growth of orange roughy (Hoplostethus atlanticus): a comparison of annulus counts with radiometric ageing. Canadian Journal of Fisheries Aquatic Science 52(2): 391-401.
Von Bertalanffy L. 1938. A quantitative theory of organic growth (inquiries on growth laws II). Human Biology 10(2): 181-213.

Zale AV \& SG Merrifield. 1989. Species profiles: life histories and environmental requirements of coastal fishes and invertebrates (South Florida)-ladyfish and tarpon, 17 pp. U.S. Fish and Wildlife Service, Luisiana.

Zar JH. 1999. Biostatistical analysis, 718 pp. Prentice Hall, Upper Saddle River.

Zerbi A, C Aliaume \& JC Joyeux. 2001. Growth of juvenile tarpon in Puerto Rican estuaries. Bulletin of Marine Science 64(1): 9-19.

Recibido el 31 de octubre de 2013 y aceptado el 23 de julio de 2014

Editor Asociado: Mauricio Landaeta D. 